\title{
CONTROLLING THE DYNAMICS OF BURGERS EQUATION WITH A HIGH-ORDER NONLINEARITY
}

\author{
NEJIB SMAOUI
}

Received 7 April 2004

\begin{abstract}
We investigate analytically as well as numerically Burgers equation with a high-order nonlinearity (i.e., $u_{t}=v u_{x x}-u^{n} u_{x}+m u+h(x)$ ). We show existence of an absorbing ball in $L^{2}[0,1]$ and uniqueness of steady state solutions for all integer $n \geq 1$. Then, we use an adaptive nonlinear boundary controller to show that it guarantees global asymptotic stability in time and convergence of the solution to the trivial solution. Numerical results using Chebychev collocation method with backward Euler time stepping scheme are presented for both the controlled and the uncontrolled equations illustrating the performance of the controller and supporting the analytical results.
\end{abstract}

2000 Mathematics Subject Classification: 35B37, 35Q53, 93D21.

1. Introduction. The viscous Burgers equation

$$
\frac{\partial v}{\partial t}=v \frac{\partial^{2} v}{\partial x^{2}}-2 v \frac{\partial v}{\partial x}
$$

introduced by Burgers [4, 5] as a simple model for a turbulent flow through a channel, has received a lot of attention in recent years from both the mathematical and control communities [1, 2, 3, 6, 7, 8, 9, 10, 11, 14, 15, 16, 18, 19, 20, 21]. In [4], Burgers proposed a mathematical model for turbulence given by

$$
\begin{aligned}
L \frac{d U}{d t} & =P-\frac{v U}{L}-\frac{1}{L} \int_{0}^{L} v^{2} d x, \\
\frac{\partial v}{\partial t} & =v \frac{\partial^{2} v}{\partial x^{2}}-2 v \frac{\partial v}{\partial x}+\frac{U}{L} v,
\end{aligned}
$$

where $U$ and $v$ are velocities connected with the primary and secondary motions, respectively. The quantities $P$ and $v$ are positive constants representing the external force and the kinematic viscosity, respectively. The space variable $x$ is the coordinate in the direction of the cross dimension of the channel and extends from 0 to $L$. When $v$ differs from zero, it is said that there is turbulence in the system.

If the turbulence is not activated by energy transmission from a primary motion (i.e., $U=0$ ), (1.3) simplifies to the viscous Burgers equation (1.1). However, when there is a constant transmission of energy from the primary motion to the secondary motion (i.e., $U \neq 0$ ), Burgers equations simplify to the original Burgers equation given by

$$
\frac{\partial v}{\partial t}=v \frac{\partial^{2} v}{\partial x^{2}}-2 v \frac{\partial v}{\partial x}+\frac{1}{L} U v
$$


Equation (1.4) can be easily transformed to the generalized Burgers equation,

$$
\frac{\partial u}{\partial t}=v \frac{\partial^{2} u}{\partial x^{2}}-u \frac{\partial u}{\partial x}+m u+h(x)
$$

where $h(x)$ is a forcing term. The cases $m=0$ and $m=1$ in (1.5) subject to periodic boundary conditions were analyzed by the author $[19,20]$ and the author and Nicolaenko [21]. In [19, 21], the existence of inertial manifold was established through the use of Kwak transformation [17], and in [20], adaptive and nonadaptive nonlinear boundary control of the generalized Burgers equation was investigated.

In this paper, we consider Burgers equation,

$$
\frac{\partial u}{\partial t}=v \frac{\partial^{2} u}{\partial x^{2}}-u^{n} \frac{\partial u}{\partial x}+m u+h(x)
$$

subject to Dirichlet boundary conditions on $[0,1]$ and with a high-order nonlinearity, that is, when $n$ is an integer greater than or equal to $1, m \in \mathbb{R}$, and $h \in L^{2}(0,1)$. It should be noted that (1.6) was also used to model traffic flows for different values of $n$ (see Haberman [13], and Farlow [12]). Furthermore, using an adaptive nonlinear boundary control, we are able to control the dynamics of (1.6) subject to a generalized form of boundary conditions.

2. Existence of an absorbing ball in $L^{2}$. In this section, we show that (1.6) with Dirichlet boundary conditions admits an absorbing ball in $L^{2}(0,1)$, and show uniqueness of steady state solutions for all integers $n \geq 1$.

Proposition 2.1. Let $m<v / 2$, and let $v \in \mathbb{R}^{+}$be the viscocity, $u_{0} \in L^{2}(0,1)$ be the initial condition, and $h \in L^{2}(0,1)$ be the forcing term. Every generalized solution to

$$
u_{t}=v u_{x x}-u^{n} u_{x}+m u+h(x) ; \quad 0<x<1,
$$

subject to

$$
u(0, t)=u(1, t)=0
$$

satisfies the inequality

$$
\|u\| \leq \sqrt{\frac{2}{v(v-2 m)}}\|h\|, \quad \forall t \geq t_{0}
$$

with

$$
t_{0}=\frac{1}{v-2 m} \ln \left(\frac{v(v-2 m)\left\|u_{0}\right\|^{2}}{\|h\|^{2}}+1\right) .
$$

Proof. Multiplying (2.1) by $u$ and integrating, we obtain

$$
\frac{d}{d t}\left(\frac{1}{2} \int_{0}^{1} u^{2} d x\right)=v \int_{0}^{1} u u_{x x} d x-\int_{0}^{1} u^{n+1} u_{x} d x+m \int_{0}^{1} u^{2} d x+\int_{0}^{1} u h d x
$$


The term $\int_{0}^{1} u^{n+1} u_{x} d x=\int_{0}^{1}\left(u^{n+2} /(n+2)\right)_{x} d x=0$, since $u(0, t)=u(1, t)=0$. Therefore, (2.5) becomes

$$
\frac{d}{d t}\left(\frac{1}{2} \int_{0}^{1} u^{2} d x\right)=v \int_{0}^{1} u u_{x x} d x+\int_{0}^{1} m u^{2} d x+\int_{0}^{1} u h d x .
$$

Integrating by parts the term $\int_{0}^{1} u u_{x x} d x,(2.6)$ becomes

$$
\frac{d}{d t}\left(\frac{1}{2} \int_{0}^{1} u^{2} d x\right)=-v \int_{0}^{1} u_{x}^{2} d x+\int_{0}^{1} m u^{2} d x+\int_{0}^{1} u h d x .
$$

Using Poincaré inequality on (2.7), we get

$$
\frac{d}{d t}\left(\frac{1}{2} \int_{0}^{1} u^{2} d x\right) \leq(m-v) \int_{0}^{1} u^{2} d x+\int_{0}^{1} \sqrt{v} u \frac{1}{\sqrt{v}} h d x .
$$

Using Cauchy-Schwartz inequality on (2.8), we get

$$
\frac{d}{d t}\left(\frac{1}{2} \int_{0}^{1} u^{2} d x\right)+(v-m) \int_{0}^{1} u^{2} d x \leq\left(\int_{0}^{1} v u^{2} d x\right)^{1 / 2}\left(\int_{0}^{1} \frac{1}{v} h^{2} d x\right)^{1 / 2}
$$

Using Young's inequality, we obtain

$$
\frac{1}{2} \frac{d}{d t}\|u\|^{2}+(v-m)\|u\|^{2} \leq \frac{v}{2}\|u\|^{2}+\frac{1}{2 v}\|h\|^{2}
$$

or

$$
\frac{d}{d t}\|u\|^{2}+(v-2 m)\|u\|^{2} \leq \frac{1}{v}\|h\|^{2} .
$$

Now, applying Grownwall's inequality on (2.11), we obtain

$$
\|u\|^{2} \leq e^{-(v-2 m) t}\left\|u_{0}\right\|^{2}+\int_{0}^{t} \frac{1}{v}\|h\|^{2} e^{-(v-2 m)(t-s)} d s .
$$

Since

$$
\int_{0}^{t} \frac{1}{v}\|h\|^{2} e^{-(v-2 m)(t-s)} d s=\frac{1}{v(v-2 m)}\|h\|^{2}\left(1-e^{-(v-2 m) t}\right),
$$

equation (2.12) becomes

$$
\|u\|^{2} \leq e^{-(v-2 m) t}\left\|u_{0}\right\|^{2}+\frac{1}{v(v-2 m)}\left(1-e^{-(v-2 m) t}\right)\|h\|^{2} .
$$

Given $\left\|u_{0}\right\|^{2}$, if we choose $t \geq t_{0}$ with

$$
t_{0}=\frac{1}{(v-2 m)} \ln \left(\frac{v(v-2 m)\left\|u_{0}\right\|^{2}}{\|h\|^{2}}+1\right),
$$

then

$$
\|u\|<\sqrt{\frac{2}{v(v-2 m)}}\|h\|
$$


It follows from (2.16) that (1.6) admits an absorbing ball in $L^{2}(0,1)$.

Proposition 2.2. Let $u^{s}$ be the steady state solution or the attractor of (1.6) with Dirichlet's boundary conditions, then $u^{s}$ satisfies the following inequalities:

(a)

$$
\left\|u^{s}\right\| \leq \frac{1}{\sqrt{v(v-2 m)}}\|h\|
$$

(b)

$$
\left\|u_{x}^{s}\right\| \leq \frac{1}{v-m}\|h\|
$$

Proof. (a) Since $u^{s}$ is a steady state solution, then from (2.11),

$$
(v-2 m)\left\|u^{s}\right\|^{2} \leq \frac{1}{v}\|h\|^{2},
$$

or

$$
\left\|u^{s}\right\|^{2} \leq \frac{1}{v(v-2 m)}\|h\|^{2}
$$

Therefore,

$$
\left\|u^{s}\right\| \leq \frac{1}{\sqrt{v(v-2 m)}}\|h\|
$$

To prove part (b), we know from (2.7) that the steady state solution satisfies

$$
v \int_{0}^{1}\left(u_{x}^{s}\right)^{2} d x=m \int_{0}^{1}\left(u^{s}\right)^{2} d x+\int_{0}^{1} u^{s} h d x
$$

Using the Poincaré inequality and the Cauchy-Schwartz inequality on (2.22), we get

$$
(v-m)\left\|u_{x}^{s}\right\|^{2} \leq\left\|u^{s}\right\| \cdot\|h\| .
$$

Finally, using the Poincaré inequality on (2.23), we obtain the desired result.

THEOREM 2.3. Let $n=1$. Then, the steady state solution of (1.6), satisfying the Dirichlet boundary conditions, is unique if $m<v / 2$ and $\|h\|<(2 / 3)(v-m)^{2}$.

Proof. We will prove the case for $n=1$; the case $n \geq 2$ is similar. Suppose there are two steady state solutions or attractors $u$ and $v$ such that

$$
\begin{gathered}
v u_{x x}-u u_{x}+m u+h(x)=0, \\
v v_{x x}-v v_{x}+m v+h(x)=0 .
\end{gathered}
$$

Let $w=u-v$. Then,

$$
v w_{x x}-u w_{x}-v_{x} w+m w=0
$$


Multiplying the above equation by $w$, integrating from 0 to 1 , and using the Dirichlet boundary conditions leads to

$$
v \int_{0}^{1} w_{x}^{2} d x+\int_{0}^{1} u\left(\frac{w^{2}}{2}\right)_{x} d x+\int_{0}^{1} v_{x} w^{2} d x-m \int_{0}^{1} w^{2} d x=0 .
$$

Again, using integration by parts on $\int_{0}^{1} u\left(w^{2} / 2\right)_{x} d x$, we obtain

$$
v \int_{0}^{1} w_{x}^{2} d x-\int_{0}^{1} w^{2}\left(\frac{u_{x}}{2}-v_{x}+m\right) d x=0 .
$$

Equation (2.27) can be written as

$$
v\left\|w_{x}\right\|^{2} \leq\left\|w^{2}\right\|\left(\frac{1}{2}\left\|u_{x}\right\|+\left\|v_{x}\right\|+m\right) .
$$

Now, by using part (b) of Proposition 2.2, we obtain

$$
v\left\|w_{x}\right\|^{2} \leq\left\|w^{2}\right\|\left(\frac{1}{2(v-m)}\|h\|+\frac{1}{v-m}\|h\|+m\right)
$$

or

$$
\left\|w_{x}\right\|^{2} \leq\left\|w^{2}\right\|\left(\frac{3}{2 v(v-m)}\|h\|+\frac{m}{v}\right)
$$

Since

$$
\left\|w^{2}\right\|_{L^{2}(0,1)} \leq\|w\|_{L^{\infty}(0,1)}^{2} \leq\left\|w_{x}\right\|_{L^{2}(0,1)}^{2}
$$

it follows that

$$
\left\|w_{x}\right\|^{2} \leq\left(\frac{3}{2 v(v-m)}\|h\|+\frac{m}{v}\right)\left\|w_{x}\right\|^{2} .
$$

If

$$
\frac{3}{2 v(v-m)}\|h\|+\frac{m}{v}<1
$$

or

$$
\|h\|<\frac{2}{3}(v-m)^{2},
$$

then $w=w_{x}=0$, which implies $u=v$.

3. Controlling the dynamics. In this section, we construct an adaptive regulator design for Burgers equation with a high-order nonlinearity,

$$
\frac{\partial u}{\partial t}=v \frac{\partial^{2} u}{\partial x^{2}}-u^{n} \frac{\partial u}{\partial x}+m u
$$


subject to the following boundary conditions:

$$
\begin{aligned}
& a \frac{\partial u}{\partial x}(0, t)+b u(0, t)=u_{1}(t), \\
& c \frac{\partial u}{\partial x}(1, t)+d u(1, t)=u_{2}(t),
\end{aligned}
$$

where $a, b, c$, and $d$ are arbitrary constants. It should be noted that the boundary conditions used in this section are general, and can be reduced to Neumann, Dirichlet, or Robin conditions. Before showing the global asymptotic stability of (3.1) and (3.2), we first prove the following lemmas.

LEMMA 3.1. Let

$$
F(t):=\int_{0}^{t} e^{\beta(t-\tau)} f(\tau) d \tau, \quad \beta<0 .
$$

If $f \in L^{1}(0, \infty)$, then $F(t) \rightarrow 0$ as $t \rightarrow \infty$.

Proof.

$$
|F(t)| \leq e^{\beta t / 2}\|f\|_{L^{1}}+\int_{t / 2}^{\infty}|f(\tau)| d \tau
$$

LEMmA 3.2. Let $\beta<0$ and $n \in \mathbb{N}$. For any $u(0, t) \in L^{2}(0, \infty) \cap L^{2(n+1)}(0, \infty)$,

(a) $\int_{0}^{t} e^{\beta(t-\tau)}|u(0, \tau)| d \tau \rightarrow 0$ as $t \rightarrow \infty$,

(b) $\int_{0}^{t} e^{\beta(t-\tau)}\left|u^{n+2}(0, \tau)\right| d \tau \rightarrow 0$ as $t \rightarrow \infty$.

Proof. We use Cauchy-Schwartz inequality and Lemma 3.1 to proof this lemma.

$$
\begin{aligned}
\int_{0}^{t} e^{\beta(t-\tau)}|u(0, \tau)| d \tau & \leq\left(\int_{0}^{t} e^{\beta(t-\tau)} d \tau\right)^{1 / 2}\left(\int_{0}^{t} e^{\beta(t-\tau)} u^{2}(0, \tau) d \tau\right)^{1 / 2} \\
& \leq \sqrt{\frac{-1}{\beta}}\left(\int_{0}^{t} e^{\beta(t-\tau)} u^{2}(0, \tau) d \tau\right)^{1 / 2}
\end{aligned}
$$

Using Lemma 3.1, part (a) is obtained. To prove part (b),

$$
\begin{aligned}
& \int_{0}^{t} e^{\beta(t-\tau)}\left|u^{n+2}(0, \tau)\right| d \tau \\
& \quad \leq\left(\int_{0}^{t} e^{\beta(t-\tau)}\left|u^{2}(0, \tau)\right| d \tau\right)^{1 / 2}\left(\int_{0}^{t} e^{\beta(t-\tau)}\left|u^{2(n+1)}(0, \tau)\right| d \tau\right)^{1 / 2} .
\end{aligned}
$$

Thus, if $u(0, t) \in L^{2}(0, \infty) \cap L^{2(n+1)}(0, \infty)$, the desired result is obtained.

THEOREM 3.3. Let $m<v / 2$ and let $n \geq 1$ be an integer. Let $u(x, t)$ be a solution of (3.1) with arbitrary initial conditions in $L^{2}(0,1)$ and satisfying the boundary conditions (3.2), where $a, b, c$, and $d$ are arbitrary constants and $a \neq 0, c \neq 0$. If $u(0, t), u(1, t)$ are locally existing in $L^{2 n+2}(0, \infty)$ and the control functions $u_{1}, u_{2}$ are given by

$$
\begin{aligned}
& u_{1}(t)=k_{1}(t) u^{2 n+1}(0, t)+k_{2}(t) u^{n+1}(0, t)+k_{3}(t) u(0, t), \\
& u_{2}(t)=k_{4}(t) u^{2 n+1}(1, t)+k_{5}(t) u^{n+1}(1, t)+k_{6}(t) u(1, t),
\end{aligned}
$$


where $k_{n}(t), n=1, \ldots, 6$, are bounded for any $t \geq 0$ with

$$
\begin{array}{ll}
\dot{k}_{1}(t)=r_{1} u^{2 n+2}(0, t), & r_{1}>0, \\
\dot{k}_{2}(t)=r_{2} u^{n+2}(0, t), & r_{2}>0, \\
\dot{k}_{3}(t)=r_{3} u^{2}(0, t), & r_{3}>0, \\
\dot{k}_{4}(t)=-r_{4} u^{2 n+2}(1, t), & r_{4}>0, \\
\dot{k}_{5}(t)=-r_{5} u^{n+2}(1, t), & r_{5}>0, \\
\dot{k}_{6}(t)=-r_{6} u^{2}(1, t), & r_{6}>0,
\end{array}
$$

then

$$
\|u(\cdot, t)\| \longrightarrow 0 \text { as } t \rightarrow \infty
$$

Proof. Let

$$
V(t)=\frac{1}{2} \int_{0}^{1} u^{2}(x, t) d x
$$

Taking the time derivative of $V(t)$, we obtain

$$
\begin{aligned}
\dot{V}(t) & =\frac{\partial}{\partial t}\left(\frac{1}{2} \int_{0}^{1} u^{2}(x, t) d x\right)=\frac{1}{2} \int_{0}^{1} 2 u(x, t) u_{t}(x, t) d x \\
& =\int_{0}^{1} u(x, t)\left\{v u_{x x}(x, t)-u^{n}(x, t) u_{x}(x, t)+m u(x, t)\right\} d x \\
& =v \int_{0}^{1} u(x, t) u_{x x}(x, t) d x-\int_{0}^{1}\left(\frac{1}{n+2} u^{n+2}(x, t)\right)_{x} d x+m \int_{0}^{1} u^{2}(x, t) d x .
\end{aligned}
$$

Using integration by parts on the first term of the right-hand side of (3.11), we get

$$
\begin{aligned}
\dot{V}(t)= & v u(1, t) u_{x}(1, t)-v u(0, t) u_{x}(0, t)-\frac{1}{n+2} u^{n+2}(1, t) \\
& +\frac{1}{n+2} u^{n+2}(0, t)+m \int_{0}^{1} u^{2}(x, t) d x-v \int_{0}^{1} u_{x}^{2}(x, t) d x .
\end{aligned}
$$

Also, using the Poincaré inequality on the last term of the right-hand side of (3.12), that is,

$$
\int_{0}^{1} u^{2}(x, t) d x \leq 2 u^{2}(0, t)+2 \int_{0}^{1} u_{x}^{2}(x, t) d x
$$

we get

$$
\begin{aligned}
\dot{V}(t) \leq & \left(m-\frac{v}{2}\right) \int_{0}^{1} u^{2}(x, t) d x+v u(1, t) u_{x}(1, t)-v u(0, t) u_{x}(0, t) \\
& -\frac{1}{n+2} u^{n+2}(1, t)+\frac{1}{n+2} u^{n+2}(0, t)+v u^{2}(0, t)
\end{aligned}
$$


Now, using the boundary conditions stated in (3.2), that is,

$$
u_{x}(0, t)=\frac{1}{a}\left(u_{1}(t)-b u(0, t)\right), \quad u_{x}(1, t)=\frac{1}{c}\left(u_{2}(t)-d u(1, t)\right),
$$

and the control law illustrated in (3.7), the above inequality (3.14) becomes

$$
\begin{aligned}
\dot{V}(t) \leq & \left(m-\frac{v}{2}\right) \int_{0}^{1} u^{2}(x, t) d x+v u^{2}(0, t)-\frac{1}{n+2} u^{n+2}(1, t)+\frac{1}{n+2} u^{n+2}(0, t) \\
& -v u(0, t)\left(\frac{1}{a}\left\{k_{1}(t) u^{2 n+1}(0, t)+k_{2}(t) u^{n+1}(0, t)+k_{3}(t) u(0, t)\right\}-\frac{b}{a} u(0, t)\right) \\
& +v u(1, t)\left(\frac{1}{c}\left\{k_{4}(t) u^{2 n+1}(1, t)+k_{5}(t) u^{n+1}(1, t)+k_{6}(t) u(1, t)\right\}-\frac{d}{c} u(1, t)\right) .
\end{aligned}
$$

Therefore, $\dot{V}(t)$ can be rewritten as

$$
\begin{aligned}
\dot{V}(t) \leq & \left(m-\frac{v}{2}\right) \int_{0}^{1} u^{2}(x, t) d x-\frac{v k_{1}(t)}{a} u^{2 n+2}(0, t)-\left(\frac{v k_{2}(t)}{a}-\frac{1}{n+2}\right) u^{n+2}(0, t) \\
& -\left(\frac{v k_{3}(t)}{a}-v-\frac{v b}{a}\right) u^{2}(0, t)+\frac{v k_{4}(t)}{c} u^{2 n+2}(1, t) \\
& +\left(\frac{v k_{5}(t)}{c}-\frac{1}{n+2}\right) u^{n+2}(1, t)+\left(\frac{v k_{6}(t)}{c}-\frac{d v}{c}\right) u^{2}(1, t) .
\end{aligned}
$$

Now, we introduce a nonnegative energy function $E(t)$ as follows:

$$
\begin{aligned}
E(t)= & V(t)+\left(\frac{v}{2 a r_{1}}\right)\left(k_{1}(t)\right)^{2}+\left(\frac{a}{2 v r_{2}}\right)\left(\frac{v k_{2}(t)}{a}-\frac{1}{n+2}\right)^{2} \\
& +\left(\frac{a}{2 v r_{3}}\right)\left(\frac{v k_{3}(t)}{a}-v-\frac{v b}{a}\right)^{2}+\left(\frac{v}{2 c r_{4}}\right)\left(k_{4}(t)\right)^{2} \\
& +\left(\frac{c}{2 v r_{5}}\right)\left(\frac{v k_{5}(t)}{c}-\frac{1}{n+2}\right)^{2}+\left(\frac{c}{2 v r_{6}}\right)\left(\frac{v k_{6}(t)}{c}-\frac{d v}{c}\right)^{2} .
\end{aligned}
$$

If we evaluate the time derivative of $E(t)$, and substitute $\dot{V}(t)$ from (3.17) and $\dot{k}_{n}(t)$, $n=1, \ldots, 6$ from (3.8) into (3.18), we get

$$
\dot{E}(t) \leq\left(m-\frac{v}{2}\right) \int_{0}^{1} u^{2}(x, t) d x
$$

This implies that if $m<v / 2$, then $E(t) \leq E(0)$. Since $u(0, t)$ and $u(1, t) \in L_{l o c}^{2 n+2}(0, \infty)$, it follows from (3.8) that $k_{j}(t)$ can be defined as continuous functions on $[0, \infty)$. Then, (3.18) and (3.19) will imply that $k_{j}, j=1, \ldots, 6$ are bounded, and then (3.8) will imply that $u(i, t) \in L^{2}(0, \infty) \cap L^{2 n+2}(0, \infty)(i=0,1)$. 


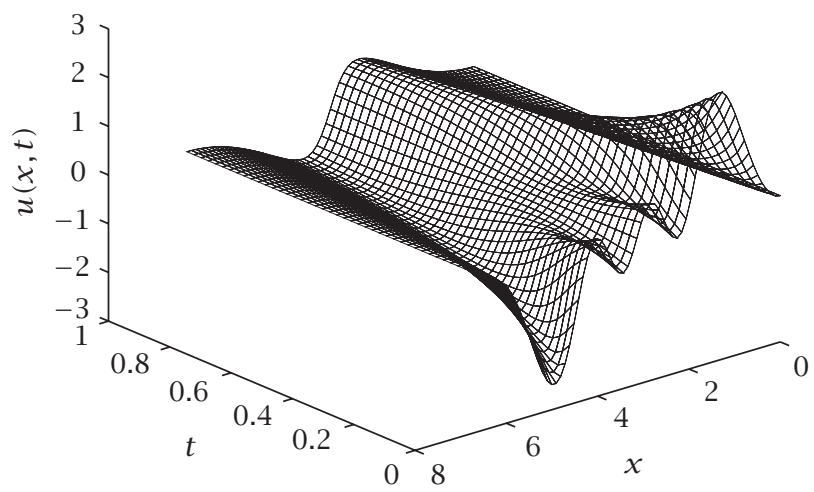

FIGURE 4.1. Time evolution of the uncontrolled Burgers equation (3.1) when $n=1, m=0.1, v=0.3$, and $(a, b, c, d)=(1,0,1,0)$.

Now, to show the global asymptotic stability of (3.1) and (3.2), we use Gronwall's inequality on (3.17):

$$
\begin{aligned}
V(t) \leq & V(0) e^{(m-v / 2) t} \\
+ & v \int_{0}^{t}\left\{\frac{-k_{1}(\tau)}{a} u^{2 n+2}(0, \tau)+\left(\frac{1}{(n+2) v}-\frac{k_{2}(\tau)}{a}\right) u^{n+2}(0, \tau)\right. \\
& \left.+\left(1+\frac{b}{a}-\frac{k_{3}(\tau)}{a}\right) u^{2}(0, \tau)\right\} e^{(m-v / 2)(t-\tau)} d \tau \\
+ & v \int_{0}^{t}\left\{\frac{k_{4}(\tau)}{c} u^{2 n+2}(1, \tau)+\left(\frac{k_{5}(\tau)}{c}-\frac{1}{(n+2) v}\right) u^{n+2}(1, \tau)\right. \\
& \left.+\left(\frac{k_{6}(\tau)}{c}-\frac{d}{c}\right) u^{2}(1, \tau)\right\} e^{(m-v / 2)(t-\tau)} d \tau .
\end{aligned}
$$

Then, using Lemmas 3.1 and 3.2, one can deduce that

$$
\|u\| \longrightarrow 0 \text { as } t \longrightarrow \infty
$$

4. Numerical results. If we express the discrete solution of (3.1) as the Chebychev series,

$$
u^{N}(x, t)=\sum_{k=0}^{N} \hat{u}_{k}(t) T_{k}(x)
$$

where $\left\{T_{k}(x)\right\}_{k=0}^{N}$ are the Chebychev polynomials, then the discretization of (3.1) and (3.2) is

$$
\left.\frac{\partial u^{N}}{\partial t}\right|_{x=x_{j}}=v \frac{\partial^{2} u^{N}}{\partial x^{2}}-\left(u^{N}\right)^{n} \frac{\partial u^{N}}{\partial x}+\left.m u^{N}\right|_{x=x_{j}}, \quad j=1, \ldots, N-1,
$$




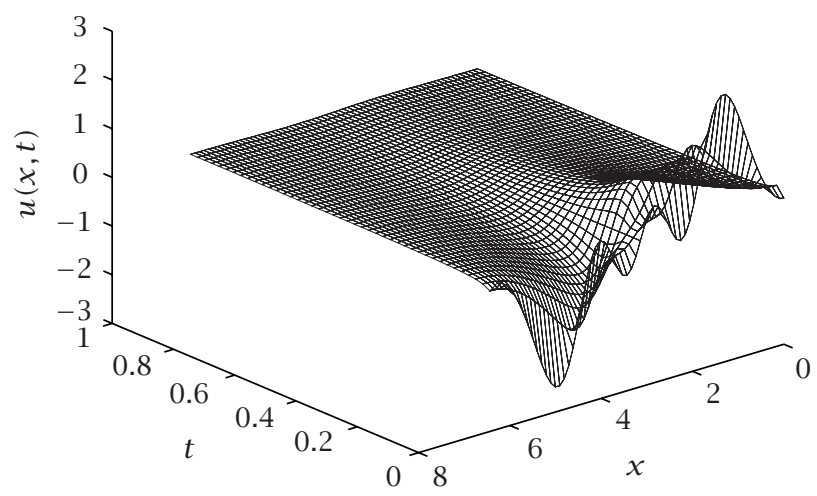

FIGURE 4.2. Time evolution of the controlled Burgers equation (3.1) when $n=$ $1, m=0.1, v=0.3,(a, b, c, d)=(1,0,1,0)$, and $r_{j}=10, j=1, \ldots, 6$.

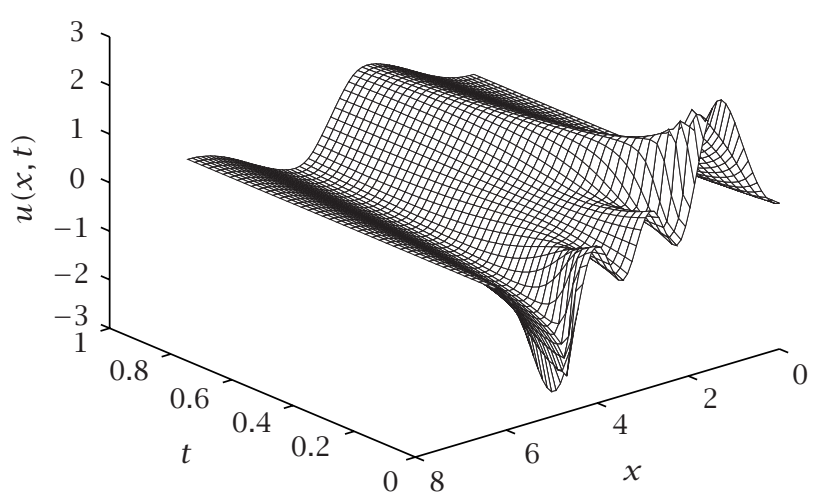

FIgURE 4.3. Time evolution of the uncontrolled Burgers equation (3.1) when $n=3, m=0.1, v=0.3$, and $(a, b, c, d)=(1,0,1,0)$.

with

$$
\begin{gathered}
a \frac{\partial u^{N}}{\partial x}(0, t)+b u^{N}(0, t)=u_{1}(t), \\
c \frac{\partial u^{N}}{\partial x}(1, t)+d u^{N}(1, t)=u_{2}(t), \\
u^{N}\left(x_{j}, 0\right)=u_{0}\left(x_{j}\right), \quad j=0, \ldots, N .
\end{gathered}
$$

The purpose of our paper is not to find the best approximation scheme for our problem, but to demonstrate our theoretical results. Therefore, the Chebychev collocation method that uses backward Euler method as a temporal scheme, the Gauss-Lobatto points, and the Chebychev collocation derivative represented in matrix form are used [9]. 


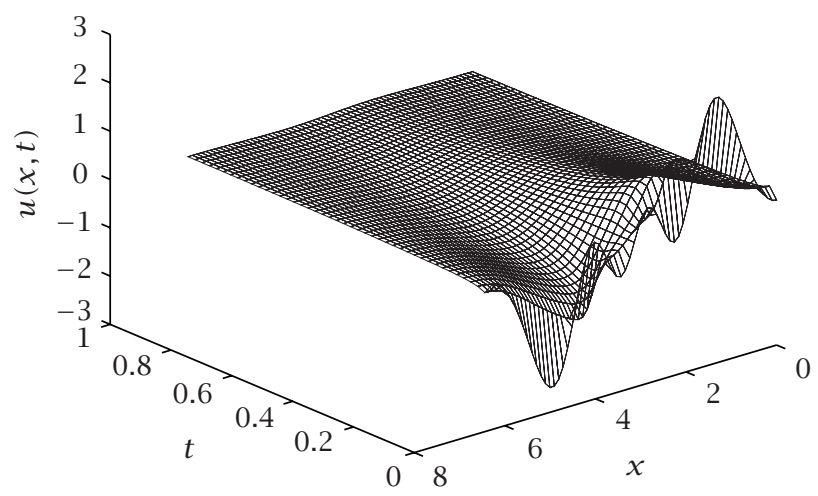

FIGURE 4.4. Time evolution of the controlled Burgers equation (3.1) when $n=$ $3, m=0.1, v=0.3,(a, b, c, d)=(1,0,1,0)$, and $r_{j}=10, j=1, \ldots, 6$.

Two computer programs that use the Chebychev collocation method with the backward Euler as a temporal scheme were written to solve (4.2) and (4.3) for both the controlled and the uncontrolled problems. For the uncontrolled problem, we choose two different values of $n$ (i.e., $n=1$ and $n=3), v=0.3, m=0.1$, and $(a, b, c, d)=(1,0,1,0)$. Figures 4.1 and 4.2 represent their corresponding solutions $u$ as it evolves in time when $u(x, 0)=\sin x+\sin 2 x+\sin 3 x$. The solution seems to converge to a nontrivial solution, although it eventually approaches zero, which could be seen only for very very large $t$, and this is in accordance with the theory presented in Section 2. In fact, for some initial data, the numerical solution gets trapped into a nontrivial steady states and never converges to zero [20]. To remedy this unsatisfactory behavior, an adaptive nonlinear boundary control, presented by (3.7), is applied to guarantee global asymptotic stability and convergence of the solution to the trivial solution as expected from the theory presented in Section 3 (see Figures 4.3 and 4.4).

ACKNOWLEDGMENT. The author would like to thank the Kuwait University for supporting this work under Project SM 02/02.

\section{REFERENCES}

[1] F. Abergel and R. Temam, On some control problems in fluid mechanics, Theor. Comput. Fluid Dyn. 1 (1990), 303-325.

[2] M. J. Ablowitz and S. De Lillo, The Burgers equation under deterministic and stochastic forcing, Phys. D 92 (1996), no. 3-4, 245-259.

[3] A. Balogh and M. Krstić, Burgers' equation with nonlinear boundary feedback: $H^{1}$ stability, well-posedness and simulation, Math. Probl. Eng. 6 (2000), no. 2-3, 189-200.

[4] J. M. Burgers, A mathematical model illustrating the theory of turbulence, Advances in Applied Mechanics, Academic Press, New York, 1948, pp. 171-199.

[5] _ The Nonlinear Diffusion Equation. Asymptotic Solutions and Statistical Problems, D. Reidel Publishing, Massachusetts, 1974.

[6] J. A. Burns and S. Kang, A control problem for Burgers' equation with bounded input/output, Nonlinear Dynam. 2 (1991), 235-262. 
[7] C. I. Byrnes, D. S. Gilliam, and V. I. Shubov, High gain limits of trajectories and attractors for a boundary controlled viscous Burgers' equation, J. Math. Systems Estim. Control 6 (1996), no. 4, 1-40.

[8] - On the global dynamics of a controlled viscous Burgers' equation, J. Dynam. Control Systems 4 (1998), no. 4, 457-519.

[9] C. Canuto, M. Y. Hussaini, A. Quarteroni, and T. A. Zang, Spectral Methods in Fluid Dynamics, Springer Series in Computational Physics, Springer-Verlag, New York, 1988.

[10] H. Choi, R. Temam, P. Moin, and J. Kim, Feedback control for unsteady flow and its application to the stochastic Burgers equation, J. Fluid Mech. 253 (1993), 509-543.

[11] A. Eden, On Burgers' original mathematical model of turbulence, Nonlinearity 3 (1990), no. 3, 557-566.

[12] S. J. Farlow, Partial Differential Equations for Scientists and Engineers, John Wiley \& Sons, New York, 1982.

[13] R. Haberman, Mathematical Models. Mechanical Vibrations, Population Dynamics, and Traffic Flow. An Introduction to Applied Mathematics, Prentice-Hall, New Jersey, 1977.

[14] K. Ito and S. Kang, A dissipative feedback control synthesis for systems arising in fluid dynamics, SIAM J. Control Optim. 32 (1994), no. 3, 831-854.

[15] T. Kobayashi, Adaptive regulator design of a viscous Burgers' system by boundary control, IMA J. Math. Control Inform. 18 (2001), no. 3, 427-437.

[16] M. Krstic, On global stabilization of Burgers' equation by boundary control, Systems Control Lett. 37 (1999), no. 3, 123-141.

[17] M. Kwak, Finite-dimensional inertial forms for the 2D Navier-Stokes equations, Indiana Univ. Math. J. 41 (1992), no. 4, 927-981.

[18] H. V. Ly, K. D. Mease, and E. S. Titi, Distributed and boundary control of the viscous Burgers' equation, Numer. Funct. Anal. Optim. 18 (1997), no. 1-2, 143-188.

[19] N. Smaoui, Analyzing the dynamics of the forced Burgers equation, J. Appl. Math. Stochastic Anal. 13 (2000), no. 3, 269-285.

[20] _ Nonlinear boundary control of the generalized Burgers equation, Nonlinear Dynam. 37 (2004), no. 1, 75-86.

[21] N. Smaoui and B. Nicolaenko, Burgers equation: Does it have an inertial manifold? Bull. Amer. Phys. Soc. 38 (1993), no. 12, 2244.

Nejib Smaoui: Department of Mathematics \& Computer Science, Kuwait University, P.O. Box 5969, Safat 13060, Kuwait

E-mail address: smaoui@mcs.sci . kuniv.edu.kw 


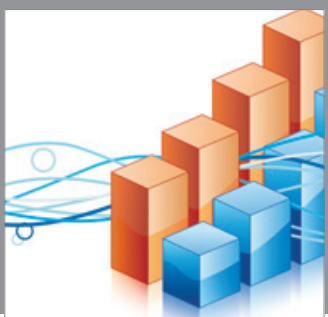

Advances in

Operations Research

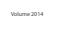

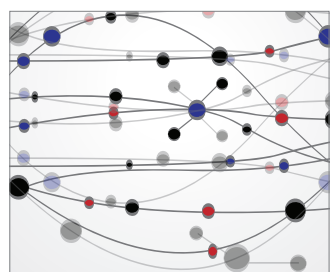

\section{The Scientific} World Journal
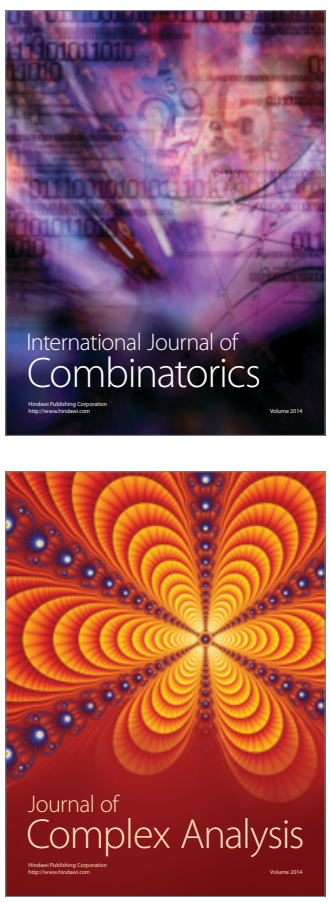

International Journal of

Mathematics and

Mathematical

Sciences
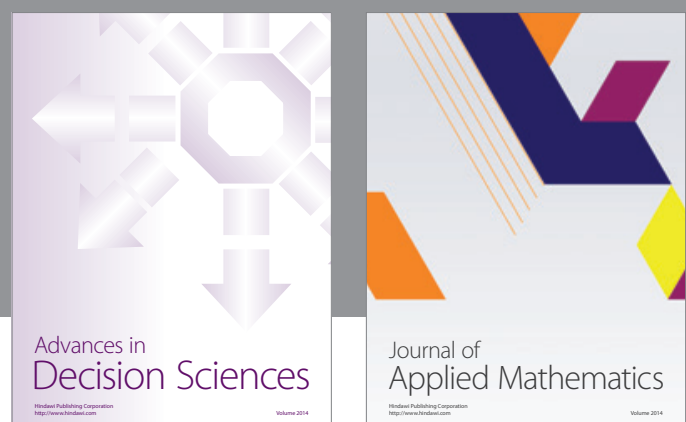

Journal of

Applied Mathematics
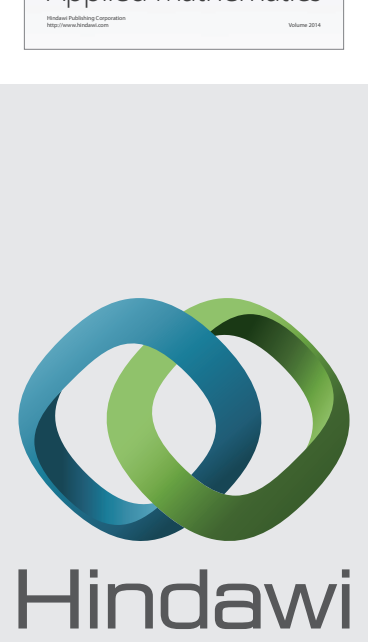

Submit your manuscripts at http://www.hindawi.com
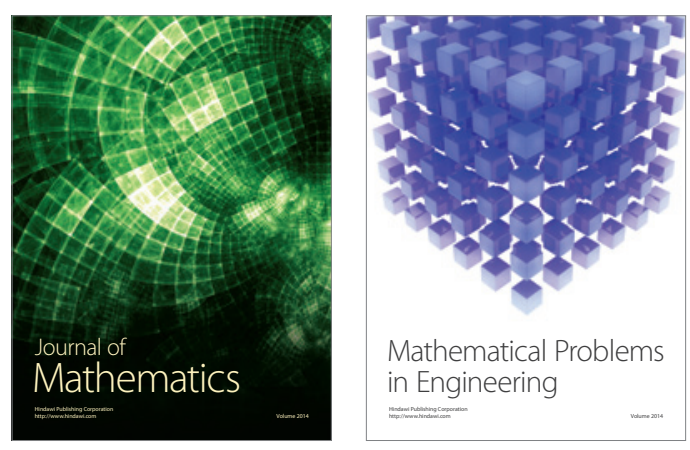

Mathematical Problems in Engineering
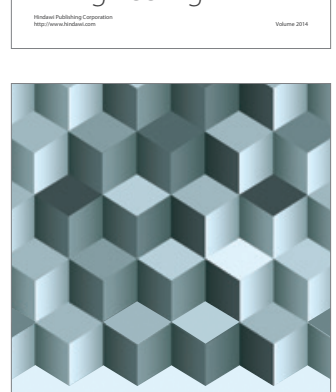

Journal of

Function Spaces
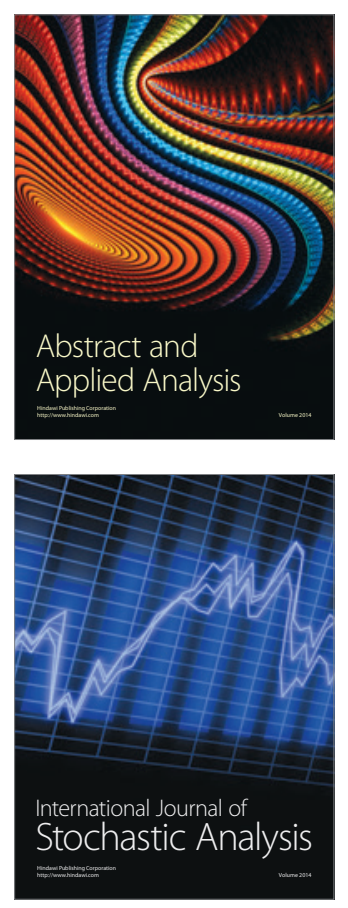

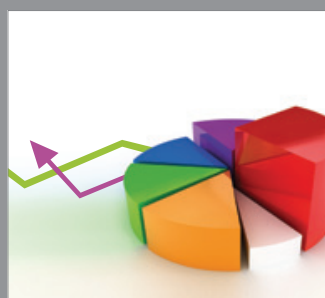

ournal of

Probability and Statistics

Promensencen
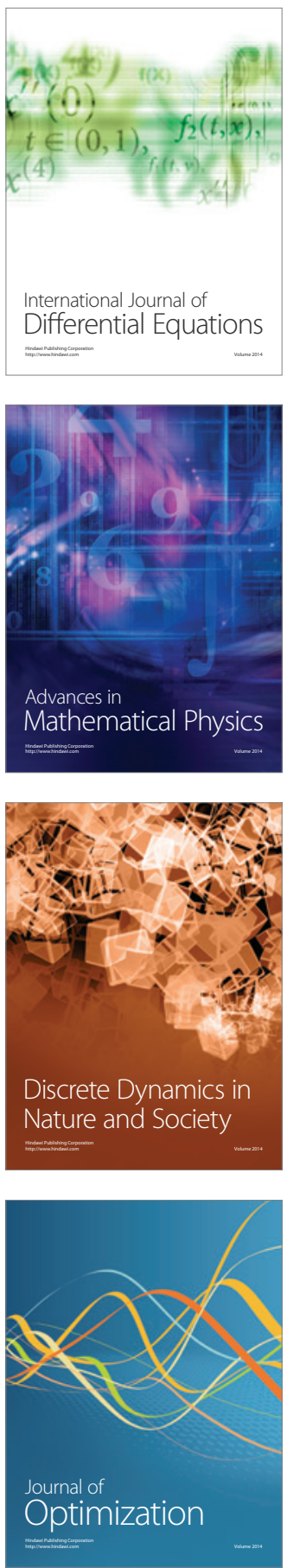